\title{
The Golden Hour: Early Interventions for Medical Emergencies during Pregnancy
}

\author{
Luis D. Pacheco, $\mathrm{MD}^{1,2}$ \\ M. J. Lozada, $\mathrm{DO}^{3}$ \\ ${ }^{1}$ Division of Maternal-Fetal Medicine, Department of Obstetrics and \\ Gynecology, The University of Texas Medical Branch, Galveston, Texas \\ 2 Division of Surgical Critical Care, Department of Anesthesiology, The \\ University of Texas Medical Branch, Galveston, Texas \\ ${ }^{3}$ Department of Anesthesiology, Vanderbilt University Medical \\ Center, Nashville, Tennessee
}

Am J Perinatol 2022;39:930-936.

\begin{abstract}
Maternal mortality has increased in the last decades in the United States as a result of increased prevalence of coexisting medical diseases such as hypertension, diabetes, and both acquired and congenital heart diseases. Obstetricians and maternal-fetal medicine physicians should have the basic medical knowledge to initiate appropriate diagnostic and early therapeutic interventions since they may be the only provider available at the time of presentation. The goal of this article is not to extensively discuss the management of complex medical diseases during pregnancy, rather we provide a concise review of key early medical interventions that will likely result in improved clinical outcomes.
\end{abstract}

\section{Key Points}

- Obstetricians and maternal-fetal medicine physicians must be familiar with initial basic management of common medical emergencies.

- Management of these complex cases is ideally multidisciplinary.

- Residency/fellowship programs should include common disease management to improve maternal outcomes.

Contrary to other developed nations, maternal mortality has not decreased and may have even increased over time in the United States. ${ }^{1}$ The rise in the prevalence of coexisting cardiovascular disease and obesity, among others, has changed the field of obstetrics and maternal-fetal medicine (MFM) dramatically. Unfortunately, most obstetrical programs do not provide adequate training in management of acute maternal care; as a result, obstetricians (OB) and MFM specialists must usually rely on multiple consultants in managing common medical emergencies during pregnancy. While multidisciplinary management of complex medical conditions is optimal, it often falls on the obstetrical team of providers to make the correct diagnosis and manage the patient before the arrival of consultant services. This initial interaction with the obstetrical service, including arriving at a differential diagnosis that will inform further evaluation and management, is critical for determining outcomes. The obstetrical service is therefore the first line of defense when patients present with lifethreatening conditions. As such, OB and MFM physicians should have basic medical knowledge to initiate such evaluation and interventions since they may be the only provider available initially. The goal of this article is not to extensively discuss management of complex medical diseases during pregnancy, rather we provide a concise review of key early medical interventions that will likely result in improved clinical outcomes. The goal for the obstetrician is to arrive at the appropriate differential diagnosis and institute the initial phase of management until the appropriate consultants arrive.

\section{Sepsis}

Sepsis is defined as the presence of an infectious process leading to a dysregulated inflammatory response resulting in received

September 18, 2020

accepted after revision

October 26, 2020

published online

November 26, 2020 (c) 2020. Thieme. All rights reserved.

Thieme Medical Publishers, Inc., 333 Seventh Avenue, 18th Floor, New York, NY 10001, USA
DOI https://doi.org/ 10.1055/s-0040-1721393. ISSN 0735-1631. 
acute organ dysfunction. ${ }^{2}$ Sepsis should be suspected in any patient with an infectious process and evidence of any organ dysfunction (e.g., confusion, hypotension, oliguria, dyspnea and hypoxemia, ileus, abnormal coagulation studies, and elevated liver enzymes). Early source control is fundamental and achievable in many cases (e.g., delivery for chorioamnionitis, dilation and curettage for retained infected placental products, and wound debridement as indicated). Together with early source control, other vital interventions include obtaining cultures as indicated (e.g., blood, urine, wounds, and vaginal) and serum lactate. ${ }^{3}$ Lactate levels should be followed during early resuscitation; a progressive decline in lactate correlates with decreased mortality. ${ }^{3}$ Early administration of broad-spectrum antibiotics (in most cases providing coverage against anaerobic and gram-positive and gramnegative bacteria), together with fluid resuscitation with 1 to $2 \mathrm{~L}$ of crystalloid (preferably lactated Ringer's solution or plasma-lyte A) aiming for a mean arterial blood pressure (MAP) of $65 \mathrm{~mm} \mathrm{Hg}$, should not be delayed. If hypotension and hypoperfusion persist, norepinephrine $(0.05 \mu \mathrm{g} / \mathrm{kg} / \mathrm{min}-$ ute as a starting dose) should be initiated targeting a MAP of $65 \mathrm{~mm} \mathrm{Hg} .{ }^{3,4}$ During the midtrimester, physiologic vasodilation may result in MAP below $65 \mathrm{~mm} \mathrm{Hg}$; the latter should not be the sole indication for aggressive fluid resuscitation and vasopressor support in the absence of other markers of hypoperfusion such as oliguria, confusion, tachycardia, cold extremities, and elevated serum lactate.

\section{Ischemic Stroke}

Ischemic stroke should be suspected with the acute onset of neurologic deficits, such as paresthesia, hemiparesis or hemiplegia, seizures, dysarthria, facial droop, or confusion/ obtundation, among others. Once suspected, stroke protocols should be activated immediately. Most hospitals have such a protocol or have a process for emergency consultation of a stroke team. Obstetrical care providers should be familiar with their particular institutional approaches. Imaging with computed tomography (without contrast, followed by angiography in many centers) should procced without delay, as this will identify optimal candidates for reperfusion therapy. ${ }^{5}$ Early measures to prevent secondary brain injury must be undertaken as soon as possible. If the airway cannot be protected, the patient should be immediately intubated, aiming for a normal arterial partial pressure of oxygen (above $80 \mathrm{~mm} \mathrm{Hg}$ ) and carbon dioxide (35-45 mm Hg in nonpregnant patients, $30 \mathrm{~mm} \mathrm{Hg}$ in pregnancy). Hypotonic fluids (e.g., the lactated Ringer's solution or plasma-lyte A) should be avoided, as they will result in hyponatremia, potentially worsening cerebral edema. ${ }^{5}$ Normal saline should be the initial fluid utilized. Hyperglycemia and hypoglycemia should be avoided, targeting glucose levels between 140 and $180 \mathrm{mg} / \mathrm{dL}{ }^{5}$ Any temperature elevation should be managed as soon as possible since hyperthermia worsens neuronal injury. Cooling blankets targeting normothermia are indicated.

If the patient is a candidate for reperfusion with systemic tissue plasminogen activator (tPA; window between presentation and onset of symptoms of 4.5 hours or less), blood pressure control is important. In nonpregnant individuals, treatment is required if the blood pressure is above $185 / 110 \mathrm{~mm}$ Hg prior to administering tPA. ${ }^{6}$ For tPA recipients during pregnancy, we recommend targeting blood pressures between 140 and $160 / 90$ and $110 \mathrm{~mm} \mathrm{Hg}$ with the use of labetalol (sequential boluses of 10, 20,40, and $80 \mathrm{mg}$ at every 10 minutes with a maximum cumulative dose of $300 \mathrm{mg}$ ) or nicardipine (continuous infusion starting at $2.5 \mathrm{mg} / \mathrm{h}$ with increments of $2.5 \mathrm{mg}$ every $5-10$ minutes to a maximum of $15 \mathrm{mg} / \mathrm{h})^{5}$ The window for endovascular intervention with local thrombolysis and clot stent retrieval of proximal thrombi is up to 24 hours after onset of symptoms. ${ }^{7}$ For this procedure, we recommend targeting the same blood pressures described for systemic tPA. If tPA is administered, aspirin and deep vein thrombosis (DVT) prophylaxis should not be started until 24 hours later and after documenting the absence of hemorrhagic transformation post tPA.

If no tPA or thrombectomy is planned, blood pressure should also be maintained between 140 and 160/90 and $110 \mathrm{~mm} \mathrm{Hg}$. In these patients, aspirin 160 to $300 \mathrm{mg} /$ day, together with DVT prophylaxis, should be started as soon as possible. ${ }^{8}$

\section{Hemorrhagic Stroke}

Hemorrhagic strokes from uncontrolled hypertension commonly affect the basal ganglia. The clinical presentation of a hemorrhagic stroke may be similar to the ischemic counterpart; suspect it with any acute onset of neurologic dysfunction including seizures, paresthesia, hemiparesis or hemiplegia, dysarthria, facial droop, or confusion/obtundation. A hypertensive response usually follows the stroke event in an attempt to increase cerebral perfusion pressure. Current guidelines recommend blood pressure treatment with values above 220/120 mm Hg. ${ }^{6}$ Treatment aiming for lower blood pressures (systolic blood pressure $<140 \mathrm{~mm} \mathrm{Hg}$ ) does not improve neurologic outcomes and may increase the risk of end organ damage (acute kidney injury). ${ }^{9}$ During pregnancy, we recommend maintaining blood pressure between 140 and 160/90 and $110 \mathrm{~mm} \mathrm{Hg}$ with labetalol or nicardipine as described above. $^{5}$ As with ischemic strokes, prevention of secondary brain injury is paramount: avoid hypotonic fluids, fever, and hypo/hyperglycemia (target glucose: $140-180 \mathrm{mg} / \mathrm{dL}$ ). If the patient is on low-dose aspirin, discontinuation is appropriate; transfusion of platelets, however, is not indicated unless a neurosurgical procedure is planned. ${ }^{10}$ Thrombocytopenia may result in hematoma expansion. Most experts recommend maintaining a platelet count above $100,00 / \mathrm{mm}^{3}$ with platelet transfusions as needed. ${ }^{11}$ Reversal of anticoagulants, such as warfarin (with intravenous [IV] vitamin $\mathrm{K}$ and prothrombin complex concentrates), unfractionated heparin (with protamine sulfate), and the new direct oral anticoagulants (with idarucizumab or andexanet $\alpha$ ) may be required. ${ }^{10}$ Intubation with mechanical ventilation may be necessary if the patient is unable to protect her airway or if hypoxemia and/or hypercapnia develop (as the latter two result in cerebral vasodilation with worsening intracranial hypertension). If preeclampsia is suspected in the differential diagnosis, magnesium sulfate should be administered. 


\section{Aortic Dissection}

The clinical presentation of aortic dissection typically includes sudden onset of severe chest pain which radiates to the interscapular region. Risk factors for aortic dissection include Ehlers-Danlos, Marfan's, and Turner's syndromes; fibromuscular dysplasia; and congenital bicuspid aortic valve. If suspected, the most important early medical management goal is rapid blood pressure control, as uncontrolled hypertension will propagate the dissection. Treatment with antihypertensives should begin as soon as possible, even before imaging, if clinical suspicion is high. Vasodilators should not be administered prior to adequate $\beta$ blockade since the acute decrease in afterload will increase shear stress with further dissection. ${ }^{12}$ Instead, treatment is started with IV esmolol (25-300 $\mu \mathrm{g} / \mathrm{kg} /$ minute) followed by an IV vasodilator, such as nicardipine $(2.5-15 \mathrm{mg} / \mathrm{h})$ or nitroprusside $(0.2-2 \mu \mathrm{g} / \mathrm{kg} / \mathrm{min})$, aiming for a systolic blood pressure below $120 \mathrm{~mm} \mathrm{Hg} .{ }^{6}$ Alternatively, IV labetalol monotherapy may be utilized, as it is a combined $\alpha$ - and $\beta$-blockers. Proximal aortic dissection (Stanford's A) requires surgical repair. Most distal dissections (Stanford's B) are managed expectantly.

\section{Myocardial Infarction}

Myocardial infarction (MI) usually presents with sudden onset of chest pain which radiates to the upper extremities and/or jaw. Other symptoms include diaphoresis, dyspnea, nausea, vomiting, and epigastric pain. Women are prone to atypical presentations including lack of significant chest pain. Grossly, MI may be divided into ST MI (elevation of the ST segment) and non-ST MI (without elevation of the ST segment). Elevation of serum troponins reflects myocardial necrosis. Unlike in nonpregnant individuals, most MI during pregnancy are due to coronary dissection (especially during the third trimester and postpartum period). ${ }^{13}$ Most coronary dissections affect the left descending coronary artery.

Initial management of a suspected MI includes pain relief with nitroglycerin (0.4-mg sublingual in every 5 minutes up to three doses) or morphine (2-4 mg IV). Oxygen should be administered if peripheral oxygen saturation $\left(\mathrm{SpaO}_{2}\right)$ is below $90 \% .{ }^{14}$ The cornerstone of management is administration of concomitant anticoagulation and antiplatelet therapy. IV unfractionated heparin (UFH) should be started (bolus of 60 units $/ \mathrm{kg}$, maximum 4,000 units, followed by an initial infusion of 12 units $/ \mathrm{kg} / \mathrm{h}$, maximum 1,000 units/h, aiming for an activated partial thromboplastin time of 50-70 seconds) together with chewed noncoated aspirin $(162-325 \mathrm{mg}$, followed by $81 \mathrm{mg}$ daily). ${ }^{15}$ Dual antiplatelet therapy is standard and consists of adding a P2Y12 blocker, such as clopidogrel, ticagrelor, or prasugrel, to aspirin. Available limited data suggest that clopidogrel $(300 \mathrm{mg}$ oral load followed by $75 \mathrm{mg}$ a day) is safe in pregnancy. ${ }^{16}$ No data exist on the safety of ticagrelor or prasugrel during pregnancy. Recent studies suggest that ticagrelor is the preferred agent for patients treated conservatively, while prasugrel is optimal among patients undergoing percutaneous coronary intervention (PCI). ${ }^{17}$ If in doubt, addition of a P2Y12 blocker may be delayed until the coronary angiography is performed (if no PCI is indicated, then clopidogrel or ticagrelor may be added; if PCI is indicated, then prasugrel will be ideal). ${ }^{17}$ If hypertension and tachycardia are present, IV $\beta$-blockers are indicated (metoprolol $2.5-5 \mathrm{mg}$ at every 5 minutes to a maximum of $15 \mathrm{mg}$ or esmolol $25-300 \mu \mathrm{g} / \mathrm{kg} / \mathrm{min}$ ). Persistent hypertension may be treated with IV nitroglycerin 5 to $100 \mu \mathrm{g} / \mathrm{min}$ (avoid if right ventricular MI is suspected). If coronary angiography reveals a coronary dissection, patients are commonly managed conservatively without PCI, as coronary interventions may worsen the dissection. ${ }^{18} \mathrm{UFH}$ should be stopped after the diagnosis of coronary dissection, and continuation of aspirin and $\beta$-blockers is recommended. ${ }^{18}$ Methergine is contraindicated in patients with acute MI.

\section{Seizures (Noneclamptic)}

Most generalized tonic-clonic epileptic seizures are selflimited. During the episode, administration of oxygen and positioning the patient in the left lateral decubitus is recommended. First-line medication is IV lorazepam (0.1 $\mathrm{mg} / \mathrm{kg}$, usually starting with $4 \mathrm{mg}) .^{19}$ If IV access is not available, intramuscular (IM) midazolam (10 mg) is equally effective. ${ }^{20}$ Most patients will require a long acting antiepileptic drug (AED) concomitantly. Commonly used agents include IV phenytoin or phosphenytoin $(15-20 \mathrm{mg} / \mathrm{kg}$ ) or levetiracetam (500-2,000 mg IV). In patients previously receiving a specific AED, a loading dose of the maintenance agent is commonly indicated. Refractory cases may require endotracheal intubation followed by infusions of midazolam (preferred) or propofol. ${ }^{21}$

\section{Acute Right Ventricular Failure}

Acute right ventricular failure (RV failure) may occur with any condition that increases right ventricular afterload (e.g., pulmonary embolism, air embolism, amniotic fluid embolism, or exacerbation of existing pulmonary hypertension by triggers such as hypoxemia, acidosis, or hypercarbia). Clinically, patients may present with systemic hypotension, dyspnea, oxygen desaturation, peripheral edema, hepatic congestion, and distended jugular veins. The diagnosis may be easily confirmed with limited bedside transthoracic echocardiography (TTE; - Fig. 1). The most important aspect of acute treatment is to avoid fluids. Overzealous fluid administration worsens hypotension by further dilating the RV, with left-sided displacement of the interventricular septum resulting in left ventricular obliteration and decreased cardiac output. ${ }^{22}$ Instead, hypotension should be treated with vasopressors (IV norepinephrine starting at 0.05 $\mu \mathrm{g} / \mathrm{kg} / \mathrm{min}$ and titrated to a MAP of $65 \mathrm{~mm} \mathrm{Hg}$ ). Oxygen should be administered if hypoxemia is present since the latter worsens RV failure by causing pulmonary vasoconstriction. ${ }^{22}$ Contractility of the RV may be improved by starting an inotropic agent, such as dobutamine $(2.5-5 \mu \mathrm{g} / \mathrm{kg} / \mathrm{min})$ or milrinone $(0.25-0.75 \mu \mathrm{g} / \mathrm{kg} / \mathrm{min})$. If pulmonary embolism $(\mathrm{PE})$ is the underlying cause, immediate therapeutic anticoagulation with UFH should be started ( $80 \mathrm{U} / \mathrm{kg}$ bolus, followed by $18 \mathrm{U} / \mathrm{kg} / \mathrm{h}$, aiming for an activated partial thromboplastin time of $60-85$ seconds or an anti-Xa value of $0.3-0.7 \mathrm{U} / \mathrm{mL}$ ). 


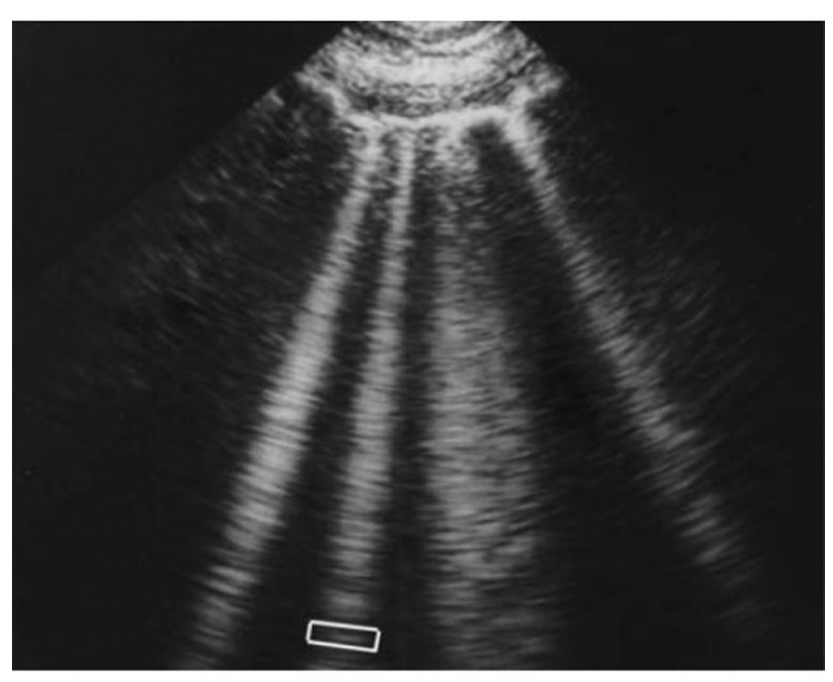

Fig. 1 Acute right ventricular failure. Please note the massively dilated right ventricle compressing the left ventricle. Reproduced with permission under Creative Commons license type BY 2.0 from Jochmann et al. ${ }^{31}$

The presence of hypotension (systolic blood pressure below $90 \mathrm{~mm} \mathrm{Hg}$ ) in the setting of PE warrants the use of thrombolytic therapy, such as tPA. ${ }^{23}$ For massive PE, the recommended dose of tPA is $100 \mathrm{mg}$ IV over 2 hours. ${ }^{23}$ Thrombolysis should not be delayed if indicated during pregnancy. ${ }^{24}$ Recent surgery (e.g., cesarean section) is a relative contraindication to using tPA, but its use in this life-threatening condition may be indicated even in the setting of a recent surgical intervention.

\section{Acute Left Ventricular Failure}

Acute left ventricular failure commonly presents with new onset dyspnea, hypoxemia, tachypnea, and orthopnea. Clinical examination reveals bilateral pulmonary crackles. Bedside point-of-care ultrasonography will show B lines (rocket lines, -Fig. 2). ${ }^{25}$ In severe cases, decreased systolic function may result in hypotension, oliguria, confusion, and cold clammy extremities. The immediate management of acute cardiogenic pulmonary edema centers on preload and afterload reduction combined with both respiratory and inotropic support. All fluids should be discontinued. Diuresis with

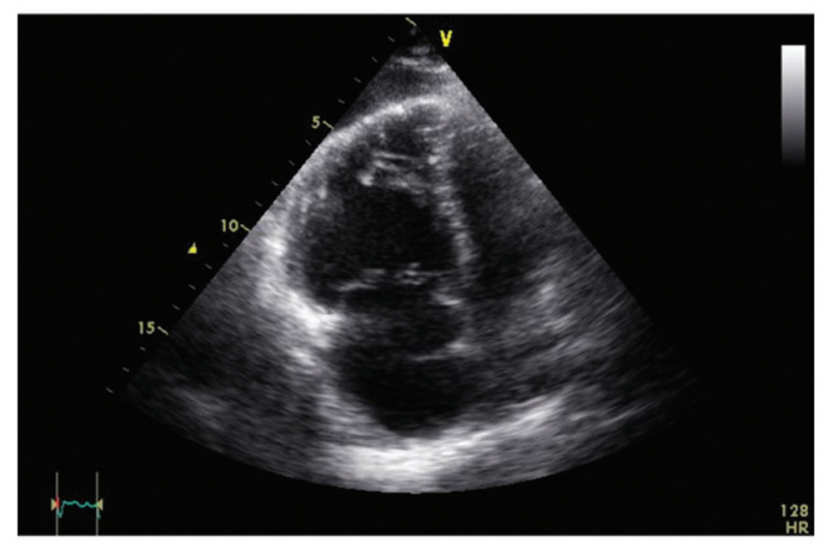

Fig. 2 Hyperechoic B lines extending to the bottom of the screen indicating pulmonary edema. Reproduced with permission under Creative Commons license type BY 4.0 from Macias. ${ }^{32}$ furosemide (intermittent boluses or a continuous infusion) is indicated. ${ }^{26}$ Common doses include 20 - to 80 -mg IV boluses at every 6 to 8 hours or an infusion of 1 to $10 \mathrm{mg} / \mathrm{h}$. Besides its diuretic effect, furosemide induces venodilation with subsequent decreased preload. The use of morphine sulfate (2$4 \mathrm{mg}$ IV) also results in venodilation and preload reduction. If systemic hypertension is present, afterload reduction is indicated. Commonly used agents in this setting include IV nitroglycerin $(5-300 \mu \mathrm{g} / \mathrm{min})$, nitroprusside $(0.2-2 \mu \mathrm{g} / \mathrm{kg} /$ $\mathrm{min})$, nicardipine $(2.5-15 \mathrm{mg} / \mathrm{h})$, and hydralazine $(5-10 \mathrm{mg}$ every 20 minutes). Nitroglycerin may be particularly effective, as it will improve pulmonary edema by inducing systemic venodilation with decreased preload. Beta-blockers should be avoided in acute decompensated left-ventricular failure. Respiratory support with noninvasive mechanical ventilation (e.g., CPAP) commonly results in improved oxygenation and improvement in pulmonary edema (a continuous positive airway pressure of $8-10 \mathrm{~cm} \mathrm{H}_{2} \mathrm{O}$ is a reasonable starting point). In patients with cardiogenic shock (systolic blood pressure below $90 \mathrm{~mm} \mathrm{Hg}$ ) and evidence of end-organ hypoperfusion, blood pressure support with norepinephrine (starting at $0.05 \mu \mathrm{g} / \mathrm{kg} / \mathrm{min}$ and titrated to a MAP of $65 \mathrm{~mm} \mathrm{Hg}$ ) is usually indicated. Addition of inotropic agents (e.g., dobutamine or milrinone) is commonly required, as norepinephrine may further decrease cardiac output after increasing systemic vascular resistances. ${ }^{26}$ Refractory cases may require mechanical support with left ventricular assist devices.

\section{Diabetic Ketoacidosis}

Diabetic ketoacidosis (DKA) usually presents with nonspecific symptoms including nausea, vomiting, abdominal pain, polyuria, and polydipsia leading to dehydration together with a gap metabolic acidosis and hyperglycemia. Other findings include deep fast breathing (Kussmaul's respiration) in an attempt to clear carbon dioxide. A fruity breath odor indicates acetone clearance. The diagnosis is usually confirmed by identifying $\beta$-hydroxy butyric acid in blood with a wide anion gap. The anion gap is calculated as (Sodium - [ chloride + bicarbonate]), and it is considered increased when above $12 \mathrm{mmol} / \mathrm{L}$. Early management of DKA includes IV fluids, insulin administration, and potassium replacement. Patients with DKA are usually volume depleted secondary to osmotic diuresis and require IV fluid resuscitation. This may be accomplished with 1 to $2 \mathrm{~L}$ of crystalloid (normal saline, lactated Ringer's solution, or plasma-lyte A) in the first 1 to 2 hours, depending on the severity of hypovolemia, followed by an infusion of 150 to $250 \mathrm{cc} / \mathrm{h}$. Once a glucose value of 200 to $250 \mathrm{mg} / \mathrm{dL}$ is reached, $5 \%$ dextrose may be added to each bag of crystalloid to prevent iatrogenic hypoglycemia. Normal saline has been associated with a higher risk of acute kidney injury secondary to hyperchloremia in critically ill patients. ${ }^{27}$ Although this has not been described in patients with DKA, if serum chloride is elevated following use of normal saline, we recommend switching to a balanced crystalloid solution, such as lactated Ringer's solution or plasma-lyte A. IV insulin should be started at a rate of 0.1 $\mathrm{U} / \mathrm{kg} / \mathrm{h} .^{28}$ If blood glucose does not decrease by at least 50 to $70 \mathrm{mg} / \mathrm{dL}$ in the first hour, the infusion rate should be 


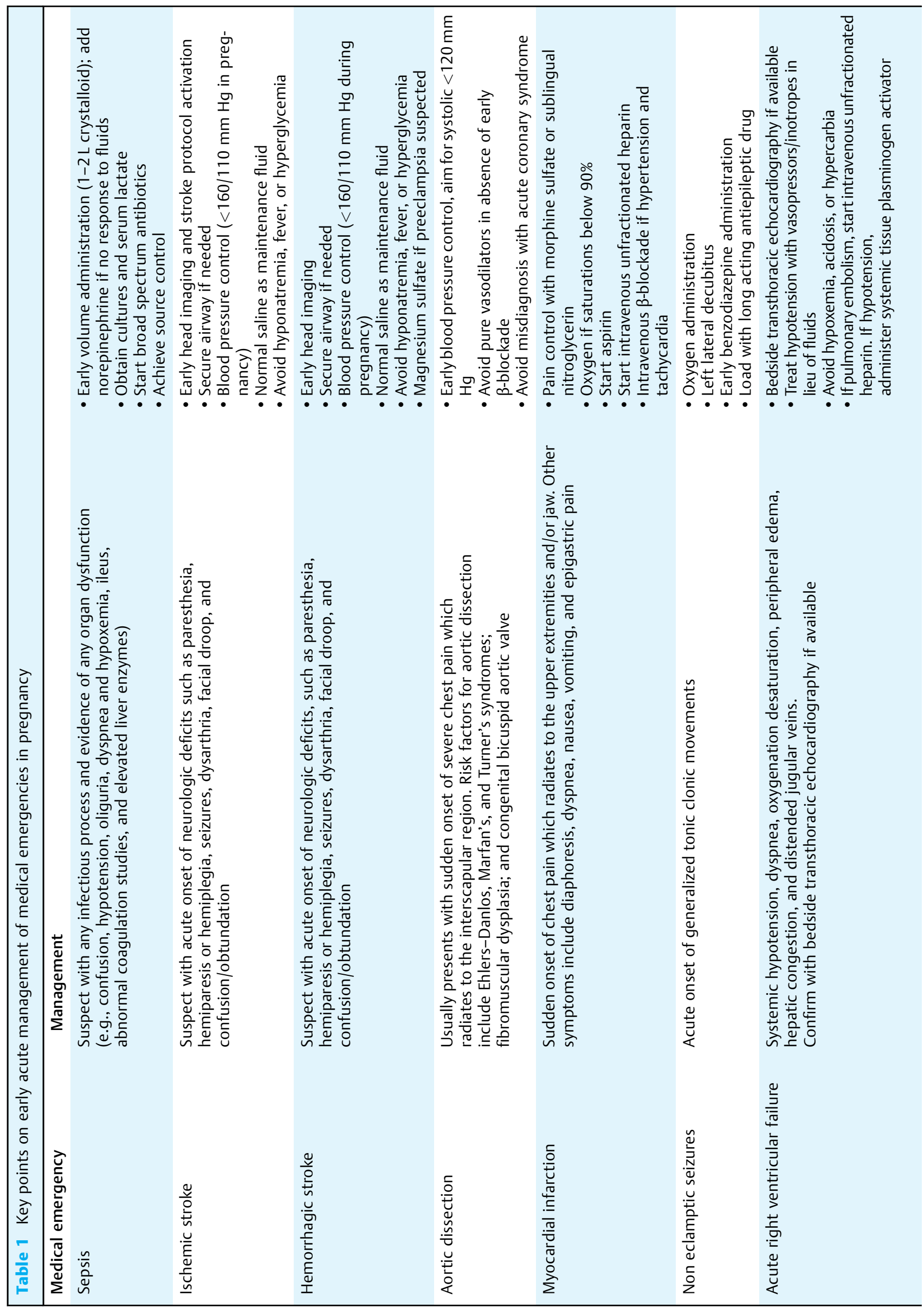




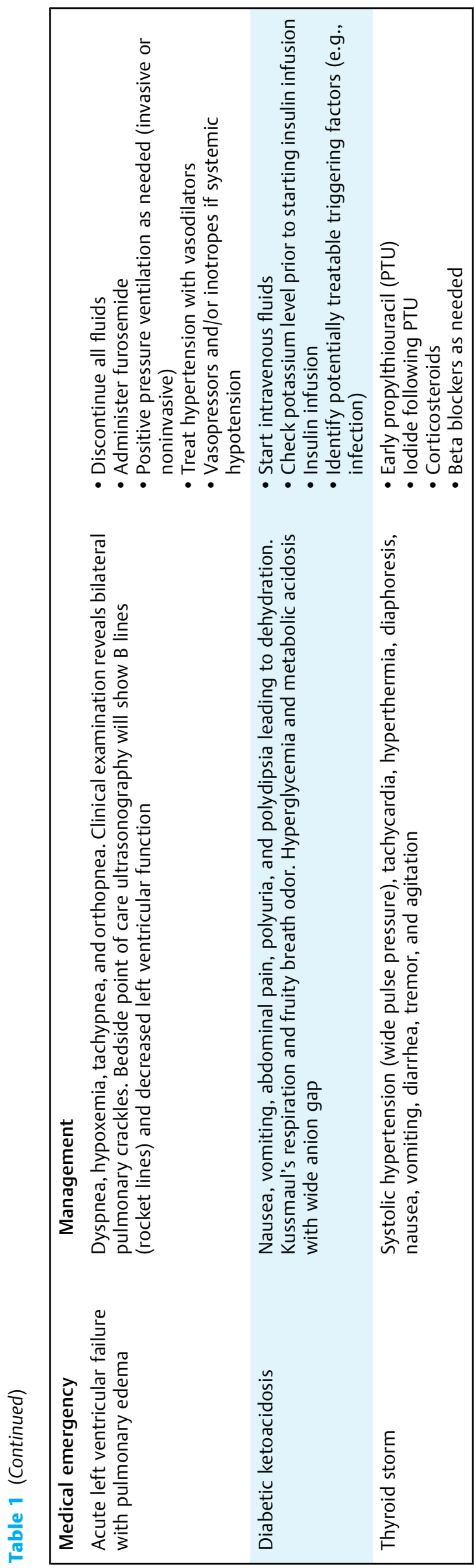

doubled. Importantly, in patients with significant hypokalemia (serum potassium $<3.3 \mathrm{mEq} / \mathrm{L}$ ), potassium replacement should be started prior to insulin to avoid the potential for insulin-induced life-threatening hypokalemia. ${ }^{28,29}$ Usually, replacement of $\mathrm{KCl} 20 \mathrm{mEq}$ IV per hour for 2 hours before insulin administration will suffice. In patients with normal serum potassium, maintenance fluids should contain potassium (20-40 mEq/L). In women presenting with potassium levels above $5.3 \mathrm{mEq} / \mathrm{L}$, initial fluids are administered without potassium; however, serial potassium measurements are indicated since hypokalemia will be "unmasked" as IV fluids and insulin are administered. Clinicians should actively seek out any treatable precipitating factor, such as infection.

Fetal heart rate anomalies, including minimal or absent variability, are frequently seen with maternal acidosis and should not lead to a rush for cesarean delivery. The latter should be reserved for prolonged bradycardia that does not resolve with maternal resuscitation. Beta mimetics should be avoided in patients with DKA.

\section{Thyroid Storm}

Thyroid storm usually presents with systolic hypertension (wide pulse pressure), tachycardia, hyperthermia, diaphoresis, tremor, nausea, vomiting, diarrhea, and agitation. Acute management is directed at blocking synthesis, release, and peripheral action of thyroid hormones. Propylthiouracil (PTU) should be started immediately at a dose of $1 \mathrm{~g}$ orally (nasogastric or orogastric tube if unable to swallow), even before the laboratory testing results are available. Once the diagnosis is confirmed, the loading dose is followed by $200 \mathrm{mg}$ at every 6 hours. $^{30}$ One hour after PTU is started and iodide is administered. Iodide may be given as potassium iodide (KI; 5 drops orally every 8 hours) or Lugol's solution (10 drops every 8 hours). IV sodium iodide is another option (1 g every 8 hours). ${ }^{30}$ Dexamethasone $2 \mathrm{mg}$ IV every 6 hours for four doses also will decrease thyroid hormone production and secretion. Hypertension and tachycardia usually require management with $\beta$-blocking agents (propranolol, labetalol, or esmolol). IV esmolol (25-300 $\mu \mathrm{g} / \mathrm{kg} / \mathrm{min})$ is particularly useful in patients at risk of high-output cardiac failure (e.g., severe tachycardia with mild pulmonary edema secondary to decreased ventricular filling time) since iatrogenic worsening of cardiac output following $\beta$-blockade is easily reversible after stopping the infusion (esmolol half-life is only a few minutes long). - Table 1 provides a summary of our recommendations.

\section{Conclusion}

Obstetrical care providers are often the first to respond to the above emergencies. In all the conditions described here, timeliness of the intervention affects outcome. OBs are therefore required to arrive at the diagnosis and initiate management in a timely manner and before any help arrives. We recognize that many of these recommendations are extrapolated from nonpregnant individuals. Future studies should ideally include pregnant women to test the efficacy in obstetric patients. - Table 1 provides a summary of our recommendations. 
Conflict of Interest

None declared.

Acknowledgment

The authors thank LeAnne Garcia in the Publication, Grant, and Media Support Office of the Department of Obstetrics and Gynecology, The University of Texas Medical Branch at Galveston, for the editing services. These services were provided free of cost as part of their affiliation with the department.

\section{References}

1 MacDorman MF, Declercq E, Cabral H, Morton C. Recent increases in the U.S. maternal mortality rate: disentangling trends from measurement issues. Obstet Gynecol 2016;128(03):447-455

2 Singer M, Deutschman CS, Seymour CW, et al. The third international consensus definitions for sepsis and septic shock (sepsis-3). JAMA 2016;315(08):801-810

3 Rhodes A, Evans LE, Alhazzani W, et al. Surviving sepsis campaign: International Guidelines for Management of Sepsis and Septic Shock: 2016. Crit Care Med 2017;45(03):486-552

4 Plante LA, Pacheco LD, Louis JMSociety for Maternal-Fetal Medicine (SMFM). Electronic address: pubs@smfm.org. SMFM consult series \#47: sepsis during pregnancy and the puerperium. Am J Obstet Gynecol 2019;220(04):B2-B10

5 Pacheco LD, Hankins GDV, Saad AF, Saade GR. Acute management of ischemic stroke during pregnancy. Obstet Gynecol 2019;133 (05):933-939

6 Whelton PK, Carey RM, Aronow WS, et al. 2017 ACC/AHA/AAPA/ $\mathrm{ABC} / \mathrm{ACPM} / \mathrm{AGS} / \mathrm{APh} / \mathrm{ASH} / \mathrm{ASPC} / \mathrm{NMA} / \mathrm{PCNA}$ Guideline for the Prevention, Detection, Evaluation, and Management of High Blood Pressure in Adults: a report of the American College of Cardiology/American Heart Association Task Force on Clinical Practice Guidelines. J Am Coll Cardiol 2018;71(19):e127-e248

7 Nogueira RG, Jadhav AP, Haussen DC, et al. DAWN Trial Investigators. Thrombectomy 6 to 24 hours after stroke with a mismatch between deficit and infarct. N Engl J Med 2018;378(01):11-21

8 Powers WJ, Rabinstein AA, Ackerson T, et al. American Heart Association Stroke Council. 2018 Guidelines for the early management of patients with acute ischemic stroke: a guideline for healthcare professionals from the American Heart Association/ American Stroke Association. Stroke 2018;49(03):e46-e110

9 Qureshi AI, Palesch YY, Barsan WG, et al. ATACH-2 Trial Investigators and the Neurological Emergency Treatment Trials Network. Intensive Blood-Pressure Lowering in Patients with Acute Cerebral Hemorrhage. N Engl J Med 2016;375(11):1033-1043

10 Frontera JA, Lewin JJ III, Rabinstein AA, et al. Guideline for reversal of antithrombotics in intracranial hemorrhage: a statement for healthcare professionals from the Neurocritical Care Society and Society of Critical Care Medicine. Neurocrit Care 2016;24(01):6-46

11 Goldstein JN, Gilson AJ. Critical care management of acute intracerebral hemorrhage. Curr Treat Options Neurol 2011;13(02): 204-216

12 Marik PE, Rivera R. Hypertensive emergencies: an update. Curr Opin Crit Care 2011;17(06):569-580

13 Tweet MS, Hayes SN, Codsi E, Gulati R, Rose CH, Best PJM. Spontaneous coronary artery dissection associated with pregnancy. J Am Coll Cardiol 2017;70(04):426-435

14 Hofmann R, James SK, Jernberg T, et al. DETO2X-SWEDEHEART Investigators. Oxygen therapy in suspected acute myocardial Infarction. N Engl J Med 2017;377(13):1240-1249
15 O'Gara PT, Kushner FG, Ascheim DD, et al. 2013 ACCF/AHA guideline for the management of ST-elevation myocardial infarction: a report of the American College of Cardiology Foundation/ American Heart Association Task Force on Practice Guidelines. J Am Coll Cardiol 2013;61(04):e78-e140

16 Reilly CR, Cuesta-Fernandez A, Kayaleh OR. Successful gestation and delivery using clopidogrel for secondary stroke prophylaxis: a case report and literature review. Arch Gynecol Obstet 2014;290 (03):591-594

17 Schüpke S, Neumann FJ, Menichelli M, et al. ISAR-REACT 5 Trial Investigators. Ticagrelor or prasugrel in patients with acute coronary syndromes. N Engl J Med 2019;381(16):1524-1534

18 Hayes SN, Kim ESH, Saw J, et al. American Heart Association Council on Peripheral Vascular Disease; Council on Clinical Cardiology; Council on Cardiovascular and Stroke Nursing; Council on Genomic and Precision Medicine; and Stroke Council. Spontaneous coronary artery dissection: current state of the science: a scientific statement from the American Heart Association. Circulation 2018;137(19):e523-e557

19 Alldredge BK, Gelb AM, Isaacs SM, et al. A comparison of lorazepam, diazepam, and placebo for the treatment of out-of-hospital status epilepticus. N Engl J Med 2001;345(09):631-637

20 Silbergleit R, Durkalski V, Lowenstein D, et al. NETT Investigators. Intramuscular versus intravenous therapy for prehospital status epilepticus. N Engl J Med 2012;366(07):591-600

21 Fernandez A, Claassen J. Refractory status epilepticus. Curr Opin Crit Care 2012;18(02):127-131

22 Vieillard-Baron A, Naeije R, Haddad F, et al. Diagnostic workup, etiologies and management of acute right ventricle failure : a state-of-the-art paper. Intensive Care Med 2018;44(06):774-790

23 Kearon C, Akl EA, Ornelas J, et al. Antithrombotic therapy for VTE disease: CHEST guideline and expert panel report. Chest 2016; 149(02):315-352

24 Gartman EJ. The use of thrombolytic therapy in pregnancy. Obstet Med 2013;6(03):105-111

25 Lichtenstein D. Lung ultrasound in the critically ill. Curr Opin Crit Care 2014;20(03):315-322

26 Yancy CW, Jessup M, Bozkurt B, et al. 2013 ACCF/AHA guideline for the management of heart failure: executive summary: a report of the American College of Cardiology Foundation/American Heart Association Task Force on practice guidelines. Circulation 2013; 128(16):1810-1852

27 Semler MW, Self WH, Wanderer JP, et al. SMART Investigators and the Pragmatic Critical Care Research Group. Balanced crystalloids versus saline in critically ill adults. N Engl J Med 2018;378(09): 829-839

28 American College of Obstetricians and Gynecologists' Committee on Practice Bulletins-Obstetrics. ACOG practice bulletin no. 201: pregestational diabetes mellitus. Obstet Gynecol 2018;132(06): e228-e248

29 Kamel KS, Schreiber M, Carlotti AP, Halperin ML. Approach to the treatment of diabetic ketoacidosis. Am J Kidney Dis 2016;68(06): 967-972

30 American College of Obstetricians and Gynecologists. Practice bulletin no. 148: thyroid disease in pregnancy. Obstet Gynecol 2015;125(04):996-1005

31 Jochmann N, Kiecker F, Borges AC, et al. Long-term therapy of interferon $\alpha$-induced pulmonary arterial hypertension with different PDE-5 inhibitors: a case report. Cardiovasc Ultrasound 2005;3:26

32 Macias M. Ultrasound leadership academy: lung ultrasound. Em Curious Accessed November 6, 2020 at: http://www.emcurious. com/blog-1/2014/11/13/ultrasound-leadership-academy-lungultrasound 